Artículo de investigación

Cuestiones de filosofía

ISSN: 0123-5095

Vol. 2 - No. 19

Julio - dciembre, año 2016

pp. $127-152$

\title{
Filosofía, matemática y paradojas: el caso de la paradoja Burali-Forti en la argumentación de Descartes sobre la existencia de Dios*
}

\author{
Philosophy, mathematics and paradoxes: the case of the \\ Burali-Forti paradox in Descartes' argument about the \\ existence of God
}

Henry Sebastián Rangel-Quiñonez
Javier Orlando Aguirre-Román***
Universidad Industrial de Santander

Colombia

Fecha de recepción: 24 de junio de 2016

Fecha de evaluación: 15 de julio de 2016

Fecha de aprobación: 15 de septiembre de 2016

* Artículo de investigación. Documento parte del trabajo monográfico necesario para optar al título de Filósofo de la Universidad Industrial de Santander. Grupo de investigación Politeia.

** Filósofo y Economista. Especialista en estadística de la Universidad Industrial de Santander. Estudiante de la maestría en Ciencias-Estadísticas de la Universidad Nacional sede Bogotá. Profesor asistente de la Universidad Industrial de Santander. hsrangelq@unal.edu.co

***Filósofo y Abogado. Doctor en Filosofía -Stony Brook University. Profesor AsociadoEscuela de Filosofía - Universidad Industrial de Santander. jaguirre@uis.edu.co 


\section{Resumen}

El presente escrito presenta las ventajas y desventajas de la formalización matemática como una herramienta para el análisis de argumentos complejos o difusos en la filosofía. De tal forma, aquí se encuentra un recorrido histórico de algunas consideraciones del papel de las matemáticas en la búsqueda del conocimiento. Posterior a ello, se muestra cómo por medio de la teoría de conjuntos y la abstracción matemática, es posible proponer una reinterpretación de algunos textos filosóficos. Para lograr este objetivo, se presenta, a manera de ejemplo, la formalización de los conceptos de "Dios", "error" y “Creación” dados por Descartes en su libro las Meditaciones de la filosofía primera, este ejercicio permite proponer una paradoja en donde se evidencia una posible falencia en la delimitación del concepto de Dios. Con todo ello, se demuestra por qué los enfoques matemáticos deben ser promovidos en los estudios filosóficos.

Palabras clave: Filosofía matemática, paradojas en Descartes, existencia de Dios, error.

\section{Summary}

This paper presents the advantages and disadvantages of mathematical formalization as a tool for analyzing complex and diffuse arguments in philosophy. So, here is a brief historical overview of the role of mathematics in the pursuit of knowledge. Following this, we show how using set theory, along with the use of mathematical abstraction, it is possible to propose a reinterpretation of the philosophical texts. To achieve this objective is presented, as an example, the formalization of the concepts of "God", "Error" and "Creation" given by Descartes in his book Meditations on First Philosophy, this exercise allows us to propose a paradox where a possible shortcoming is evident in the definition of the concept of God. As a result, it can be shown how mathematical approaches should be promoted in philosophical studies since can show problems in defining concepts or reflect problems in the argumentative logic.

Keywords: Mathematics Philosophy, paradoxes Descartes, God's existence, error. 


\section{INTRODUCCIÓN}

La revolución científica del siglo XVII marcó el inicio de la ciencia moderna la cual desarrolla la idea de un saber capaz de conocer (y controlar) la naturaleza basado en el uso del método experimental y las matemáticas. En palabras de Descartes (1981), las nociones generales que ha aprendido de su física y su filosofía

(...) Pues tales nociones me han hecho ver que pueden lograrse conocimientos muy útiles para la vida y que en lugar de esta filosofía especulativa que se enseña en las escuelas, puede encontrarse una filosofía práctica en virtud de la cual, conociendo la fuerza y las acciones del fuego, del agua, del aire, de los astros, de los cielos y de todos los cuerpos que nos rodean con tanta precisión como conocemos los diversos oficios de nuestros artesanos, podamos emplearlos de igual forma para todos aquellos usos que sean propios, convirtiéndonos por este medio en dueños y señores de la naturaleza. (p. 45)

Iniciada y aplicada en principio en las áreas de la astronomía y la física, esta idea nueva del saber verdadero, progresivamente, se va ampliando a otras áreas del conocimiento y de la vida humana. En efecto,

Las matemáticas desempeñaron un papel muy importante a lo largo de toda la revolución científica del siglo XVII, tanto por los enormes avances a los que dieron lugar (aparición delálgebra y del cálculo infinitesimal, por ejemplo), como por haber representado una alternativa metodológica al escolasticismo. La matematización de la naturaleza, junto al establecimiento de las reglas básicas del método experimental, fueron los dos pivotes sobre los que se asentó el nuevo paradigma metodológico para la investigación científica. (Echeverría, 2013, p. 77).

Un resultado posterior de este desarrollo fue la fuerte escisión entre las ciencias humanas y sociales, (filosofía, historia, sociología, antropología, literatura, entre otras) y las ciencias exactas (matemáticas, física, química, geometría, etc.). Las primeras dirigen su actuar por medio de las palabras y sus significados, y tienen como premisa, la mayoría de las veces, el indeterminismo de los fenómenos humanos. Las segundas usan como principal herramienta las matemáticas y asumen una realidad determinística factible de predicción. La muy conocida distinción diltheyniana entre Naturwissenschaften y Geisteswissenschaften constituye un ejemplo histórico - filosófico de esta separación. 
Críticos de esta distinción señalan que ésta no tiene en cuenta que, a la larga, el conocimiento humano es uno solo. Igualmente, se suele apuntar, esta distinción aparentemente "neutra" esconde realmente una subvaloración de las llamadas "ciencias humanas", las cuales son consideradas como "ciencias blandas", en contraposición con las "ciencias duras", que son las "ciencias naturales"1.

Un elemento que se suele olvidar en este debate es que esta distinción, elaborada sistemáticamente porprimera vez por Dilthey, fue una reacción histórica a una trayectoria que buscaba reducir todo el conocimiento humano a un modelo matemático. La definición de Hobbes de razonamiento es paradigmática en este sentido:

Por razonamiento entiendo la computación. Y computar es hallar la suma de varias cosas añadidas o conocer lo que queda cuando de una cosa se quita otra. Por lo tanto, razonar es lo mismo que sumar y restar, y si alguien añade a esto multiplicar y dividir, no estoy de acuerdo ya que la multiplicación es la suma de cosas iguales, y la división la resta de cosas iguales cuantas veces se pueda hacer. Por lo tanto todo razonamiento se reduce a estas dos operaciones de la mente: la suma y la resta (Hobbes, 2009, p. 39)2.

1 Las ciencias sociales vendrían a ser una especie de campo intermedio. Al respecto, sin embargo, se puede consultar el ensayo del filósofo alemán Jürgen Habermas titulado "Ciencias sociales reconstructivas vs. Comprensivas" (1985). En este texto Habermas reflexiona sobre los elementos comunes de las llamadas "ciencias sociales" y las llamadas "ciencias humanas". Naturalmente también puede consultarse el libro de Habermas, Conocimiento e interés (1982) proyecto que, aunque abandonado posteriormente por Habermas, contiene análisis muy interesantes sobre los distintos tipos de conocimiento en relación con las distintas clases de intereses humanos. Para leer debates más contemporáneos en relación con lo señalado en este párrafo se puede consultar, entre otros, The Future of the Sciences and Humanities (2002), Reinventing the Social Sciences (2004), The Politics of Method in the Human Sciences: Positivism and Its Epistemological Others (2005), "The natural vs. The human sciences: myth, methodology and ontology" (2013), etc. La lista de bibliografía científica sobre estos temas es interminable dada la complejidad y la larga historia del problema. Acá presentamos unos pocos títulos a manera de ejemplo. Igualmente, este tema, aunque subyace profundamente al tópico central de nuestro artículo, desborda los límites analíticos y reflexivos del mismo. Por lo tanto, lo presentamos solamente como contexto teórico general.

2 En todo caso, Hobbes tenía una concepción relativamente amplia de computación. En efecto, "Pero no hay que pensar que la computación, esto es, el razonamiento, tenga lugar solamente en los números como si el hombre se distinguiera de los demás animales sólo por su facultad de numerar (como se cuenta que opinaba Pitágoras) ya que se pueden sumar y restar magnitud a magnitud, cuerpo a cuerpo, tiempo a tiempo, grado a grado de calidad, concepto a concepto, proporción a proporción, oración a oración, nombre a nombre (en lo cual se contiene todo género de Filosofía)" (Hobbes, 2009, p. 39). 
Ahora bien, hay que señalar que esta equiparación entre conocimiento, realidad y matemática no es nueva en la historia de la filosofía. Los pitagóricos fueron los primeros en equiparar la sustancia con la matemática, en especial el número, así para ellos la cosmología y antropología están basados en relaciones matemáticas (Abbagnano, 1994, p.22-26). Estas relaciones se evidencian también en múltiples partes de la obra de Platón, el cual enaltece el conocimiento matemático por estar alejado de lo material. Podemos recordar en el famoso libro VII de La República los pasajes que exaltan a la matemática gracias a su cercanía con el alma y su carácter de suprasensible: "Ahora bien, toda la logística y aritmética tienen por objeto el número... Y asíresultan aptas para conducir a la verdad" (Platón,1993, p. 340), lo que ubica a la matemática en el segundo escalón en la idea del bien, precedido, exclusivamente, por la dialéctica (Platón,1993, p. 355-356).

Hay que reconocer que en Platón los elementos matemáticos, como el número, gozan del estatus de idea, por ende, de mayor realidad que los objetos físicos, contrario a Aristóteles, quien nos dice "El "ser" lo decimos en muchos sentidos" (Aristóteles, 1994, 1077b17) por lo que ve a la materia como sustancia de la realidad y, a sus abstracciones (número, forma, etc), como solo una arista de éstas. Pese a esta diferencia, Aristóteles resalta el uso de las matemáticas como el mejor medio para definir la esencia o entidad de los entes, pero la rechaza como parte de la filosofía primera, pues no habla sobre la sustancia en sí, ni por fuera de ella. En otras palabras, el mundo para Aristóteles (1994) no está conformado por elementos matemáticos ni siguen una lógica matemática.

Que [las matemáticas] no pueden existir en las cosas sensibles y que tal teoría resulta cargada de fantasía, ya quedó en las discusiones de las aporías [al señalar](a) que es imposible que dos sólidos estén a la vez en el mismo lugar y (b) que, además y por la misma razón, deberían estar también en las cosas sensibles las restantes potencias y naturalezas, sin existir ninguna de estas separadas $[. .$.$] (c) es evidente que resultaría imposible que se dividiera cuerpo$ alguno. En efecto, habría de dividirse por una superficie, y esta por una línea, y esta por un punto: conque si es imposible dividir el punto, también la línea, y si esta, también los otros. (1076 b).

Por otro lado, las matemáticas tampoco existen de forma paralela a las cosas:

(...) y es que, si existieran sólidos aparte de los sensibles, separados de estos, distintos y anteriores a los sensibles, es evidente que existirían también 
otras superficies separadas aparte de las superficies sensibles, y puntos y líneas (por la misma razón). Y si existen estos, a su vez existirán otros aparte de las superficies, las líneas y los puntos del sólido matemático (pues las cosas simples son anteriores a las compuestas; y si los cuerpos no sensibles son anteriores a los sensibles, por la misma razón habrá de admitirse que las superficies mismas, en sí, son anteriores a las que se dan en los sólidos inmóviles $\mathrm{y}$, por consiguiente, tales superficies y líneas son distintas de las que se dan juntamente con los sólidos separados: estas se dan, en efecto, a la vez que los sólidos matemáticos, mientras que aquellas son anteriores a los sólidos matemáticos). Y en tales superficies habrá, a su vez, líneas, pero anteriores a estas habrá de haber otras líneas y puntos por la misma razón. Y respecto de los puntos de estas líneas que son anteriores, habrá otros puntos anteriores respecto de los cuales ya no habrá otros anteriores. Y de este modo se produce un amontonamiento absurdo. (Aristóteles, 1994, 1076 b15).

Por lo anterior, las matemáticas existen en forma de potencia en la definición de las cosas y son las más apropiadas en cuanto que el Ente posee propiedades como: longitud, profundidad, unidad, pluralidad, etc. Todo objeto de estudio de las matemáticas de forma simple, o sea prescindiendo de la materia:

Ocurre, por otra parte, que las cosas poseen muchas propiedades que les pertenecen por sí mismas, en tanto que tales propiedades se dan en ellas, pues también el animal posee afecciones que le son propias en tanto que hombre, y en tanto que macho (a pesar de que no hay j"machok" ni j'hembrak" separados de los animales). Por consiguiente, las cosas tendrán también propiedades exclusivamente en tanto que son longitudes y en tanto que son superficies. Y en la medida en que aquello de que se ocupa una ciencia es anterior y más simple, en esa medida la ciencia tendrá mayor exactitud (pues exactitud es simplicidad). Será, por tanto, más exacta prescindiendo de la magnitud que con ella, y exacta en grado sumo si prescinde del movimiento; y si se ocupa del movimiento, será exacta en grado sumo respecto del movimiento primero: este es, en efecto, el más simple, y, de este, el uniforme (Aristóteles, 1994, 1078 a 5 - 1078 a 12).

Posterior a Aristóteles encontramos la filosofía de la Edad Media la cual margina de sus teorías a la matemática para la búsqueda de la verdad, pues Dios es único conducto y sustento de la sustancia, lo que incentiva un estudio minucioso de éste. Como muy bien lo muestra Gonzalo Serrano, la silogística aristotélica se constituyó 
en el modelo epistemológico por excelencia de la alta Edad Media, modelo rechazado fuertemente por Bacon, Descartes y Locke (Serrano, 2005).

Este rechazo fue compartido también por los principales expositores de la revolución científica, a saber, Copérnico, Kepler, Galileo y Newton quienes concibieron un universo regido por las leyes y los métodos de la matemática, distante al saber de las esencias que proponía Aristóteles y los medievales. De esta manera, la ciencia moderna no versa ya sobre la sustancia de los fenómenos, sino tan solo, sobre las cualidades de los mismos que pueden ser objetivamente controlables y cuantificables. "Ya no se trata del "qué", sino del "cómo", la ciencia galileana y posgalileana ya no indagará sobre la sustancia, sino sobre la función. (Reale y Antiseri, 1988, p. 173-174).

Como un gran protagonista de este rechazo, debemos contar al filósofo René Descartes (2009), quien llega a afirmar que el número y las matemáticas son ideas innatas, equiparadas a la idea de Dios. En sus propias palabras:

(...) percibo innumerables cosas particulares respecto a las figuras, el número, el movimiento y otras cosas semejantes, cuya verdad es tan evidente y tan acorde con mi naturaleza, que cuando las detecto, por primera vez, no me parece tanto que estoy aprendiendo algo nuevo, sino que recuerdo lo que ya antes sabía, o que me doy cuenta por vez primera de lo que ya hace tiempo estaba en mí, aunque no había dirigido antes hacía ello la atención de mi mente. (p. 150-151).

En este punto, se evidencia una cercanía al pensamiento Platónico de la matemática como reminiscencia e idea pura. Aquí se ha dado una bifurcación iniciada con Platón y afianzada en Descartes, que ha hecho pasar a la matemática de herramienta a ente creador y contenedor de verdad. De tal forma que, para Descartes, la matemática goza de un grado máximo de simplicidad que está íntimamente relacionado con el ser humano. Es así que vuelve a la matemática y la geometría parte indispensable del método de conocimiento, tal como es enunciado en El Discurso del método (p.1516) y es reafirmado en Reglas para la dirección del espíritu (p. 6).

El primer paso para esto fue encumbrar a las matemáticas como único conocimiento irrefutable y rechazar todos los demás conocimientos por ser probables y susceptibles de duda. Su principal argumento a favor de la matemática es la composición de partes simples y claras como los números y las operaciones (suma, resta, multiplicación y división). En palabras de Descartes (2011): 
En efecto, todo error en que pueden caer los hombres (...) no proviene jamás de una mala inferencia, sino sólo de que se dan por supuestas ciertas experiencias poco comprendidas, porque se establecen juicios a la ligera sin fundamento.

De lo cual se colige evidentemente por qué la aritmética y la geometría son mucho más ciertas que las demás disciplinas, a saber: porque solo ellas versan de un objeto tan puro y simple que no hace falta admitir absolutamente nada que la experiencia haya hecho incierto, sino que consisten totalmente en un conjunto de consecuencias que son deducidas por razonamiento (p. 6).

Con la anterior definición, Descartes establece el sendero para que las matemáticas sean el camino que lleve al espíritu de forma adecuada en la búsqueda del conocimiento.

La deducción es, según Descartes, “(...) todo aquello que se sigue necesariamente de otras cosas conocidas con certeza.” (Descartes, 2011, p. 9). Y, las matemáticas, por ser producto de la deducción, son conocidas con certeza y, por ende, son el fundamento de la búsqueda del conocimiento.

Pero ¿qué entiende Descartes por matemática? La matemática, en Descartes, elude los problemas del lenguaje y se centra en la medición y orden de las entidades simples y objetivas. Sobre este tema se discute en la Regla XVI tal como se evidencia a continuación:

"Y con este ardid [las matemáticas], no sólo economizaremos un gran número de palabras, sino, lo que es más importante, presentaremos los términos de la dificultad tan puros y desnudos que, sin omitir nada útil, no se encontrará nunca en ellos nada superfluo y que ocupe inútilmente la capacidad del espíritu, mientras la mente tenga que abarcar varias cosas a la vez." (Descartes, 2011, p. 62).

Por estas características ventajosas es que las matemáticas ocupan un papel fundamental en el método cartesiano:

“(...) se nota al fin que sólo aquellas cosas en que se estudia el orden y la medida se refiere a la matemática, no importa que tal medida se haya de buscar en números, figuras, astros, sonidos o cualquier otro objeto (...) (Descartes, 2011, p. 14)". 
Así pues, las matemáticas o el método matemático es el método cartesiano en sí. Pues en ordenar y medir las cosas intuidas es un buen resumen de lo propuesto por Descartes en el Discurso del método.

Siguiendo en la línea de tiempo encontraremos a Leibniz (1983) y su exaltación a las matemáticas al ser consideradas ideas innatas y puras de similar naturaleza a la idea de Dios. De esta forma, las matemáticas son el medio por el cual debe conducirse el conocimiento, pues:

Lo que más ha contribuido a impedirnos encontrar la adecuación o inadecuación entre las ideas es el mal uso de las palabras; y los matemáticos, los cuales forman sus pensamientos independientemente de los nombres y tienen la costumbre de tener ante su espíritu las ideas mismas, en lugar de los sonidos, han evitado en gran parte esa dificultad (p. 466).

Es claro el valor de la matemática para la filosofía de Leibniz ${ }^{3}$ pero, lo realmente importante es su carácter crítico y reflexivo de su uso, tal como lo deja expreso en el primer libro Nuevos ensayos sobre el entendimiento humano en el que acepta como regla buscar la demostración incluso de los axiomas (Leibniz, 1983, p. 114), retirando el dogmatismo del campo numérico.

Posteriormente, enraizada en la modernidad, encontramos la Ilustración y en ella a Immanuel Kant, tal vez el filósofo más representativo de esa era. En Kant (1998) se vislumbra la desmitificación de la matemática, evidenciada en un desligue ontológico con la idea de Dios, unida desdePlatón hasta Descartes. En este punto las matemáticas se afianzan como juicios sintéticos a priori que tienen la característica de referirse a la realidad y no tienen relación alguna trascendente y teológica:

Se podría pensar, de entrada, que la proposición $7+5=12$ es una simple proposición analítica, que se sigue, de acuerdo con el principio de contradicción, del concepto de suma de siete y cinco. Pero, si se observa más de cerca, se advierte que el concepto de suma de siete y cinco no contiene otra cosa que la unión de ambos números en uno solo, con lo cual no se piensa en absoluto cuál sea ese número único que sintetiza los dos. El concepto de doce no está todavía pensado en modo alguno al pensar simplemente

3 Recuérdese que es a este filósofo a quien se le atribuye la invención del cálculo infinitesimal de constante uso en las ciencias modernas. 
dicha unión de siete y cinco. Puedo analizar mi concepto de esa posible suma el tiempo que quiera, pero no encontraré en tal concepto el doce (p. 51-52)

Por otro lado, Dios es desterrado del mundo matemático trascendental y enviado al mundo trascendente, que traspasa los límites de la razón. Asípues, las matemáticas gozan el estatus de ser verdades autoevidentes que no provienen de la experiencia misma.

Esta "matematización" del conocimiento y de la vida humana constituye uno de los principales elementos de crítica a la modernidad, tal y como lo podemos ver, por ejemplo, en la filosofía de M. Heidegger (1985). En efecto, para Heidegger las matemáticas son un mero representar mimético, tal como lo serían la poesía en Platón, que no alcanza a concebir la verdad en su totalidad, sino algunos accidentes de ella. Para ejemplificar este punto recordemos un ejemplo dado en La pregunta por la cosa sobre la verdad por correspondencia:

(...) tomamos un papelito y anotamos la verdad: "Aquí estála tiza." Colocamos esta verdad escrita junto a la cosa cuya verdad es. Terminada la clase se abre las dos puertas, se ventila el aula, hay corrientes de aire y -supongamos- el papelito se vuela al pasillo. Un estudiante lo encuentra en su camino al corredor, y lee la frase: "aquí está la tiza.", y constata que eso es completamente falso. Por la corriente de aire la verdad se ha trasformado en falsedad (p. 30).

En tal forma que la matemática para Heidegger no es más que una herramienta que no puede generar algo nuevo ya existente: "Las $\mu \alpha \theta \eta \alpha \tau \alpha$, lo matemático, es aquello "de" las cosas, que en verdad ya conocemos; por consiguiente no es algo que extraemos de las cosas sino que, en cierto modo, llevamos con nosotros mismos" (Heidegger, 1985, p. 63).

Lo que va en oposición a la teoría Kantiana y vuelve a ubicar a la matemática como una forma de conocimiento del ser y no como el único y perfecto camino para llegar a la verdad.

Todo lo anterior sirve de preámbulo para el presente artículo, el cual, en todo caso, no pretende realizar un juicio histórico-filosófico definitivo a las relaciones entre la filosofía y la matemática. Partiremos simplemente de considerar en este escrito a las matemáticas como una herramienta que, como dijo Leibniz, tiene ventaja sobre el lenguaje al eludir ambiguiedades y problemas de interpretación. 
En todo caso, la misma debe ser usada con cuidado ya que un proceso de abstracción hecho a la ligera sesga el resultado de la investigación. Con esto en mente, presentaremos un escenario de análisis en donde la filosofía y la matemática se entrecruzan profundamente, y que no suele ser muy tenido en cuenta por los críticos de esta relación, a saber, la filosofía de las paradojas.

Las paradojas son una serie de enunciados que conforman una implicación lógica contradictoria, pueden ser diseñadas con el objetivo de controvertir un argumento o simplemente de presentar un dilema que no es fácilmente resuelto. En el campo de las matemáticas las paradojas han sido usadas para refutar la teoría de conjuntos, en especial, la definición de números "transinfinitos" elaborada por George Cantor y criticada por Bertrand Russell.

En términos generales el presente artículo busca mostrar cómo el uso de las matemáticas puede dar una reinterpretación a conceptos filosóficos. Para ello se presenta una formalización matemática del argumento de Descartes acerca de la existencia de Dios, la creación del universo y la naturaleza del error con el fin de mostrar la existencia de una paradoja al interior de la argumentación cartesiana. Con todo ello, se pretende mostrar por qué los enfoques matemáticos, en el contexto de los estudios filosóficos, permiten en ciertos casos evidenciar carencias en la definición de los conceptos y reflejar problemas en la lógica argumentativa. La estructura del texto es la siguiente. En un primer momento, se desarrolla una reflexión sobre la relación entre la filosofía y las paradojas. Posterior a ello, se presentan las posturas cartesianas de "Dios", el "error" y la "creación" dadas en la tercera y cuarta meditación del libro Meditaciones acerca de la filosofía primera y se muestra la existencia de la paradoja Burali-Forti en tal argumentación. Al final se sugieren algunas conclusiones.

\section{Filosofía y paradojas}

El interés de la filosofía por las paradojas no es un interés meramente especulativo o de entretención ${ }^{4}$. Las paradojas en general son consideradas anomalías en el lenguaje; anomalías que evidencian cierto conflicto con las opiniones que dábamos

4 Ver, entre otros, Beall, J.C. (editor) 2007, Revenge of the Liar. New Essays on the Paradox, New York: Oxford University Press; Cantini, A., "On a Russellian paradox about propositions and truth", in: G. Link 2004, 259-284; Field, H. 2008, Saving Truth from Paradox, New York: Oxford University Press; Garciadiego, A., 1992, Bertrand Russell and the Origins of Set-theoretic "Paradoxes", Basel: Birkhäuser; Kritchman, S. and 
por ciertas. Recordemos por ejemplo la famosa paradoja del mentiroso, tal vez una de las más famosas paradojas filosófica: una persona afirma: "Estoy diciendo mentiras". Si esa persona no está diciendo la verdad cuando afirma lo anterior, se sigue lógicamente que sí la está diciendo (pues efectivamente está diciendo que está diciendo mentiras). Sin embargo, si está diciendo la verdad, se sigue lógicamente que está diciendo lo que está diciendo, es decir, diciendo mentiras. En consecuencia, lo que esa persona dice es verdad si y solo si es falso lo que está diciendo. Algo que, evidentemente, es absurdo.

Los filósofos consideran que las paradojas son importantes puesto que son anomalías persistentes que nos permiten ver asuntos de gran profundidad y relevancia sobre posibles debilidades e incongruencias en nuestros esquemas conceptuales o sobre realidades filosóficas que no habían sido vistas anteriormente.

Existen por lo general dos actitudes básicas hacia las paradojas: o bien deben ser resueltas, o bien no deben ser resueltas. En el primer caso se trata de intentar salvar la verdad o nuestra idea de verdad, general o particular, de las terribles garras de las paradojas. En el segundo caso se trata de aprender de lo que nos dicen y aceptar los presupuestos de "nuestras verdades".

Los filósofos han clasificado a las paradojas de muchas formas: paradojas epistemológicas (la paradoja del examen sorpresa, la paradoja de la lotería, la paradoja de la bella durmiente, etc.), paradojas metafísicas (todas las relacionadas con el infinito por ejemplo), paradojas de conjuntos (la paradoja de Cantor, la paradoja de Russell, la paradoja del barbero, etc.), y muchas más que simplemente deberían ser clasificadas en sus propias categorías (por ejemplo las paradojas relacionadas con las decisiones democráticas, las paradojas teológicas o la muy conocida paradoja del mentiroso o la del Sorites).

En todo caso, como lo señalan Barwise y Etchemendy (1987),

Raz, R., 2011, "The surprise examination paradox and the second incompleteness theorem", Notices of the American Mathematical Society, 57: 1454-1458; McGee, V., 1991, Truth, Vagueness, and Paradox: An Essay on the Logic of Truth, Indianapolis and Cambridge: Hackett Publishing; Shen-Yuting, 1953, "The paradox of the class of all grounded sets", The Journal of Symbolic Logic, 18: 114; Simmons, K., 1993. Universality and the Liar, New York: Cambridge University Press. Uno de los libros más usados en las universidades para el estudio de este tema es el de Sainsbury, titulado Paradoxes, Cambridge University Press, 2009. 
The significance of a paradox is never the paradox itself, but what it is a symptom of. For a paradox demonstrates that our understanding of some basic concept or cluster of concepts is crucially flawed, that the concepts break down in limiting cases. And although the limiting cases may strike us as odd or unlikely, or even amusing, the flaw itself is a feature of the concepts, not the limiting cases that bring it to the fore. If the concepts are important tones, this is no laughing matter (p. 4).

Igualmente, según Barwise y Etchemendy (1987), An adequate analysis of a paradox must diagnose the source of the problem the paradox reveals, and thereby help us refine the concepts involved, making them truly coherent (p. 4). Las paradojas pueden ser definidas como una contradicción lógica, del tipo $(x \wedge \neg x)$, originadas esencialmente por la reflexividad de las clases, o sea por los conjuntos que se contienen a sí mismos. El filósofo inglés Bertrand Russell analizó estos problemas de la teoría de conjuntos y propuso su teoría de los tipos lógicos que tiene como finalidad evitar dichas antinomias. Las paradojas analizadas por Russell son: la paradoja de Cantor, la paradoja de Burali-Forti, la paradoja de las clases, la paradoja de las relaciones, la paradoja de las propiedades, la paradoja de Richard, la paradoja de König, la paradoja de Berry y la paradoja de Eubúlides (Whitehead y Russell, 1963, p. 60-65). Con este análisis Russell pudo identificar a la autorreferencia y al círculo vicioso como una constante en las paradojas analizadas. Sobre esto dice Russell:

"Cualquier colección que involucre al todo no debe ser parte de esta colección" (Whitehead y Russell, 1963, p. 37).

De ese modo, el origen de estas paradojas está íntimamente ligado a la incorrecta definición de límite de la clase problemática. La paradoja de Burali-Forti enunciada en 1897 es un buen ejemplo de ello. Burali-Forti define $\Omega$ como la serie de todos los ordinales bien ordenados, pero $\Omega+1$ debe ser predecesor de la serie $\Omega$, en caso contrario, este último no sería la serie de todos los ordinales bien ordenados. Al mismo tiempo, se conoce, por los principios de Peano, que todo número más la unidad produce un número sucesor y que nunca puede existir un mismo sucesor para dos números diferentes. De tal forma que $\Omega+1$ debe ser sucesor y predecesor de $\Omega$ al mismo tiempo, resultado contradictorio.

Una versión de esta paradoja es la paradoja del barbero, la cual plantea una situación hipotética donde existe un solo barbero en un pueblo el cual tiene como labor afeitar 
a todas las personas que no se afeitan a símismas. Ahora bien, la situación problemática se crea al tratar de responder la pregunta ¿el barbero debe afeitarse a sí mismo? Pues, si la respuesta es positiva, el contra argumento es que no debería hacerlo, ya que él debe afeitar únicamente a quien no se afeita a sí mismo. Por otro lado, si la respuesta es negativa, se le refutaría con la oración: el babero debería afeitarse, ya que el barbero afeita a todos lo que no se afeitan a sí mismos.

Para comprender los orígenes de esta paradoja, hay que recordar la paradoja del matemático ruso, Georg Cantor, en la segunda mitad del siglo XIX. Las premisas de esta paradoja son las siguientes: si S es el conjunto de todos los conjuntos, su conjunto potencia ${ }^{5} P(S)$ tendrá un número cardinal mayor; $(P(S)>S)$. Pero como $\mathrm{P}(\mathrm{S})$ es un conjunto, deberá ser miembro $\mathrm{S}$. De modo que su número cardinal sería inferior o igual de $\mathrm{S} ;(P(S)<\mathrm{S})$, resultando contradictorio.

La paradoja de Cantor fue analizada por Bertrand Rusell en los siguientes términos:

Por lo que se desprende de cuanto llevamos visto, es evidente que la teoría de los tipos se halla en situación de sortear todas las dificultades relativas al mayor número cardinal. En cada tipo se da un número cardinal que es el mayor, a saber, el número cardinal de la totalidad del tipo en cuestión, más dicho número se verá siempre superado por el cardinal del tipo siguiente, ya que, si a es cardinal de un tipo dado, el del tipo siguiente será 2 elevado a la potencia $\alpha$, que, como Cantor demostrará, será siempre mayor que $\alpha$. Y puesto que no hay medio de sumar entre sí tipos diferentes, no podremos hablar de "el número cardinal de todos los objetos sea cual fuere su tipo", ni habrá por consiguiente del número cardinal que sea el mayor de todos de manera absoluta. (Russell, citado por Rengifo, 2004, p.97).

El profesor Rengifo (2004, p. 97) nos precisa que las dificultades de estos argumentos se encuentran en la frase "el mayor número cardinal" dado que se puede interpretar como "el número cardinal de todos los objetos sea cual fuere su tipo" lo cual carecería de sentido; sin embargo, se podría reformular la idea en "el mayor número cardinal del tipo y orden n" de donde no se desprende alguna contradicción.

5 Se llama conjunto potencia al conjunto de todos los subconjuntos de un conjunto dado, y a su vez el número de todos los subconjuntos dado está determinado por, donde $n$ es el número de elementos del conjunto. (Rengifo, 2004, p. 48). 
De similar forma, encontramos una referencia de Russell a la paradoja de BuraliForti:

Si tomamos todos los ordinales de cualquier tipo dado, habrá siempre ordinales más altos en tipos superiores, sin que por lo demás nos sea posible agrupar un conjunto de ordinales cuyo tipo excediese de cualquier límite finito. Así pues, todos los ordinales de cualquier tipo podrán ser dispuestos en orden de magnitud en una serie bien ordenada, a la que corresponderá un número ordinal de tipo superior al de los ordinales integrantes de la serie. En el nuevo tipo, dicho nuevo ordinal no será el más alto. No hay en efecto, en ningún tipo, un ordinal que sea el más alto de todos los ordinales, sino que, en cada tipo, todos los ordinales lo son menos que algunos ordinales de tipo superior. Es imposible completar la serie de los ordinales, pues ello dará lugar a tipos superiores a todo límite finito que pudiéramos asignarles. (Russell, citado por Rengifo, 2004, p. 97).

Por lo cual se concluye que la expresión "el número ordinal de todos los ordinales" conlleva a una expresión paradójica cuando se interpreta como "el número ordinal de todos los ordinales cualquiera que sea su orden". Pero se puede evitar, tal antinomia, si se enuncia como "el número ordinal de todos los ordinales de orden n" excluyendo cualquier proposición problemática como lo es "n+1". Este punto es crucial para el presente texto, ya que el conjunto de las características de Dios, descrito por Descartes puede ser entendido como ese conjunto " $n$ " de cualquier orden, lo que provocará la contradicción aquí hallada. Todo esto se explicará con más detalle en el siguiente apartado.

\section{La FILOSOFÍa de Descartes y la PARAdOJa de la PERFECCión de Dios y la PROCEDENCIA DE LOS ERRORES}

El papel que la idea de Dios juega en la filosofía de Descartes ha sido objeto de grandes controversias. Para algunos Descartes está siendo completamente sincero cuando postula a Dios como su fundamento metafísico y epistemológico. Es por esto, entonces, que Descartes, más que el primer filósofo moderno, debe ser considerado el último filósofo escolástico. Para otros, en cambio, Descartes se está escondiendo. Descartes sabe muy bien las consecuencias ateas y mecanicistas de su nueva filosofía; sin embargo trata de esconderlas un poco en virtud del miedo a las represalias de la Iglesia. Descartes entonces tiene que escribir de una forma tal que oculte sus intenciones. Él escribe de forma tal que el lector cuidadoso 
reconocerá la importancia de sus ideas, pero estas serán expresadas de forma indirecta, una forma que a veces no parece decir lo que realmente quiere decir ${ }^{6}$.

El concepto de Dios en las Meditaciones cartesianas aparece de forma explícita en la siguiente frase: "Con el nombre de Dios entiendo cierta sustancia infinita, independiente, supremamente inteligente, supremamente poderosa, por quien, tanto yo mismo, como todo lo demás que existe, si algo más existe, hemos sido creados" (Descartes, 2009, p. 117). De tal manera, que Dios para Descartes (2009) es un ser al que le es propio, en acto y no en potencia (p. 119), todas las características que gozan de realidad y de existencia en este mundo, ya que de él proviene toda la existencia y realidad de los entes siendo la causa primera (2009, p. 125).

Ahora bien, elDios cartesiano es omnipotente y por tanto parecería capaz de ser imperfecto, si así lo desea (Descartes, 2009. p. 101). Podría por ejemplo ser engañador, ser finito, ser contenedor de errores y hasta crear una piedra tan pesada que ni él pudiera cargarla. Pero tal definición en potencia es contradictoria con el Dios en acto perfecto descrito por Descartes, ¿cómo es esto posible? Con lo anterior podríamos definir a Dios como a una unidad ${ }^{7}$ que, pese a ser indivisible contiene múltiples características. (Véase la Tabla 1 una representación extensiva del concepto de Dios).

6 Las dos perspectivas contemporáneas más conocidas que defienden la idea según la cual Descartes está "escondiéndose" son las de Hiram Caton, la cual puede encontrarse en los siguientes textos: "On the Interpretation of the Meditations", Man and World 3, 3 (1970): 224-245; "The Problem of Descartes' Sincerity", The Philosophical Forum 2, 3 (Spring 1971): 355-370; "The Origin of Subjectivity: An Essay on Descartes" (New Haven: Yale University Press, 1973), y "Will and Reason in Descartes' Theory of Error" The Journal of Philosophy 72, 4 (Feb. 27, 1975); 87-104. El otro autor que duda de la "sinceridad" de Descartes es Keneth Dorter (1973). Versiones anteriores de la "hipótesis de la insinceridad" se pueden encontrar en: Charles Adams, "Vie \& Oeuvres de Descartes, Étude Historique" (Paris: Leopold Cerf, 1910), 304-307) y Maxime Leroy, "Descartes, le philosophe au masque" (Paris: Les Editions Rieder, 1929), 1 : 15- 21 y 2: 17-42). Para ver otros textos sobre el papel de Dios en la filosofía de Descartes se puede consultar: Agostini (2015), Gorham (2012), DeHart (2012), Garber (2013), Abbeuzzese (2008), Bolton (2008), Crumplin (2008), Rocca (2005).

7 Descartes (2009) define a Dios como la unidad, simple e inseparable, puesto que cualquier división representa imperfección. (p. 125). 
Tabla 1. Representación extensiva del conjunto de Dios

\begin{tabular}{|c|c|}
\hline Dios de Descartes & Perfecto \\
\cline { 2 - 2 } & Suma inteligencia \\
\cline { 2 - 2 } & Benévolo \\
\cline { 2 - 2 } & $\ldots$ \\
\hline
\end{tabular}

Fuente: elaboración propia

Así pues, el concepto de Dios en Descartes podría representarse por medio de un conjunto que contenga a todas sus características. Asumamos a $\Omega_{1}$ como dicha representación, donde:

$\Omega_{1}=($ Perfecto, summa inteligencia $, \ldots, \infty]$

Luego de definir a Dios como un conjunto representado como $\Omega_{1}$ es necesario definir al error y a la creación de Dios, de tal forma que se logre dividir en partes pequeñas todo el objeto de estudio, para luego ser organizado de forma coherente para su posterior comprobación.

El error para Descartes proviene del hombre y no de Dios, ya que Dios es benévolo ${ }^{8}$ y perfecto. Por ello no duda en decir:

“(...) mientras pienso únicamente en Dios y me vuelvo por completo hacia él, no encuentro ninguna causa de error o de falsedad" (Descartes, 2009, p. 133).

Por consiguiente, explica Descartes, el error es causa del intelecto y de la voluntad (Descartes, 2009, p. 137) que se juntan para producir juicios de valor. En palabras de Descartes:

(...) ¿De dónde nacen mis errores? Pues únicamente de que, como la voluntad es más amplia que el intelecto, no la contengo dentro de los mismos límites,

8 “(...) porque en todo engaño o fraude se encuentra algo de imperfección; y, aunque poder engañar parezca un poco una demostración de inteligencia o de poder, no cabe duda de que querer engañar testifica malicia o debilidad, y que por lo tanto no le corresponde a Dios" (Descartes, 2009, p. 133). 
sino que la extiendo también a las cosas que no entiendo, al ser indiferente a ellas, con facilidad se desvía de lo verdadero y de lo bueno, y así me equivoco y peco. (Descartes, 2009, p. 141).

En este sentido el error es producto y causa del ser humano y no tiene ninguna relación con Dios.

Ahora bien, el ser humano, según Descartes (2009), hace parte de la creación de Dios en su totalidad y éste, gracias al libre albedrío, produce los errores (p. 143). Por lo cual podemos describir al ser humano como un subconjunto de la creación de Dios que contiene los errores, junto con otras características provenientes de Dios. En forma extensiva el ser humano estaría conformado por:

Tabla 2. Representación extensiva del hombre

\begin{tabular}{|c|c|}
\hline \multirow{2}{*}{ Ser Humano } & Sustancia \\
\cline { 2 - 2 } & Razón \\
\cline { 2 - 2 } & Error \\
\cline { 2 - 2 } & $\ldots$ \\
\hline
\end{tabular}

Fuente: elaboración propia

La Tabla 2 presenta al ser humano con todas las cualidades dadas por Dios más los errores producto de su mal juicio. Hay que recordar que el ser humano no es perfecto, como lo es la creación en su totalidad, así que habrá que entender la relación ser humano-creación para comprender la paradoja aquí propuesta.

El Dios de Descartes es un dios creador y benévolo que ha dado a su creación todos los atributos que él posee, pues Dios no puede haber privado a su creación de alguna facultad que "en su género no sea perfecta" (Descartes, 2009, 135). Sobre esto Descartes dice:

Se me ocurre también que, siempre que preguntamos si las obras de Dios son perfectas, no hay que considerar una criatura cualquiera por separado, sino toda la universalidad de las cosas; porque aquello que tal vez no sin razón parecería muy imperfecto si estuviese solo, es perfectísimo en cuanto tiene en el mundo carácter de parte (...) (Descartes,2009, 135). 
Con lo anterior, Descartes deja en claro que la creación de Dios es perfecta si se mira en su totalidad, tal como un reloj mecánico es perfecto en conjunto, pero imperfecto e inútil si es pensado sólo como un engrane.

De aquí que la creación de Dios tenga las mismas facultades de Dios, pero el ser humano, que hace parte de la creación de Dios, tiene la facultad creadora de errar, por consiguiente, la creación contiene, también, los errores producto del ser humano. Por ello se puede describir al conjunto de la creación de Dios como:

Tabla 3. Representación extensiva del conjunto de la creación de Dios

\begin{tabular}{|l|c|}
\hline $\begin{array}{c}\text { Creación de Dios } \\
\text { "Universo" }\end{array}$ & Perfecto \\
\cline { 2 - 2 } & Suma inteligencia \\
\cline { 2 - 2 } & Benévolo \\
\cline { 2 - 2 } & Error \\
\hline$\ldots$ \\
\hline
\end{tabular}

Fuente: elaboración propia

La Tabla 3 puede representarse en forma simbólica como:

$\Omega_{2}=\Omega_{1}+\varepsilon ; \varepsilon>0$

Donde $\Omega_{2}$ representan la creación que tiene similares atributos a Dios más $\varepsilon$ "los errores" producto del ser humano ${ }^{9}$, con todo esto se puede formular la siguiente paradoja.

Con todo lo anterior, es posible entender la paradoja aquí propuesta de los argumentos cartesianos. El primer argumento que hay que traer es la definición de

9 Hay que aclarar que el concepto de error no es erróneo por sí mismo, o sea no es un término reflexivo. De tal forma, que el error solo es un referente a todos los juicios equívocos que el hombre lleva, que como el mismo Descartes acepta: "la vida humana está sometida con frecuentes errores en cuestiones particulares, y hay que reconocer la debilidad de nuestra naturaleza" (Descartes, 2009, p. 195). 
Dios y de su creación.

Ecuación $1, \Omega_{1}=[$ Perfecto, summa inteligencia $, \ldots, \infty)$

Ecuación 2, $\Omega_{2}=[$ Perfecto, summa inteligencia, error, ..., $\infty)$

Igualando la ecuación 1 y la 2, tenemos:

Ecuación 3,

$[$ Perfecto, summa inteligencia $, \ldots, \infty)=$

[Perfecto, summa inteligencia, error, ..., $\infty$ )

Lo cual representa la similitud en características de Dios y la Creación. Ahora bien, el error hace parte de las características del ser humano y este hace parte de la creación. Lo cual produce, por transitividad, que las características del hombre también estén presentes en la Creación. Remplazando en la ecuación 3 se tiene:

Ecuación $4, \Omega_{1}=\Omega_{1}+\varepsilon$

La Ecuación 4 es contradictoria pues $\Omega_{1}$ es definido como el conjunto supremo y absoluto, por lo cual debe ser mayor a $\Omega_{1}+\varepsilon$, pero tal como lo definió Peano todo número más la unidad corresponde a un número superior, así que necesariamente $\Omega_{1}$ es menor a $\Omega_{1}+\varepsilon$. Lo que se representa como:

$\Omega_{1}>\Omega_{1}+\varepsilon ;$ Según la teoría cartesiana

$\Omega_{1}<\Omega_{1}+\varepsilon$; Según la teoría de conjuntos

De ello, se puede aseverar que de la definición de Dios y de error se desprende una contradicción, similar a la planteada por Burali-Forti a los números transinfinitos de Cantor.

Ahora bien, la anterior paradoja carecería de sentido si los errores son iguales a cero o negativos $(\varepsilon \leq 0)$ pues el conjunto de Dios sería igual al de la creación o mayor. Pero, ¿será que los errores pueden ser cero o negativos realmente? La respuesta es "no" en tanto que el ser humano exista; pues es indudable que el ser humano trasciende sus pensamientos a juicios, que la mayor parte de las veces 
están errados. No basta con pensar en una quimera para crear un error, pero basta con atribuirle a ella una causa para originar una imperfección y añadirle (no restarle) un accidente a la creación.

\section{A MODO DE CONCLUSIÓN}

Uno de los objetivos del texto fue mostrar que las matemáticas, al ser consideradas como un medio para llegar al conocimiento, pueden facilitar la detección de algunos problemas del lenguaje oral y escrito. Naturalmente hay muchos casos en los que esto simplemente no es posible, o solo es posible con ciertos límites. Sin embargo, hay muchos otros en los que el modelo matemático del conocimiento es de gran utilidad. En términos generales este fue el gran descubrimiento de Descartes, descubrimiento que se encuentra en los orígenes de la filosofía, el pensamiento y, en general, la sociedad moderna.

En el caso de este texto el razonamiento matemático facilitó, en la argumentación de Descartes sobre la existencia de Dios y la naturaleza del error, el descubrimiento de una paradoja formalizada que señala límites difusos para el conjunto de la creación, el cual aparece unas veces mayor al conjunto de Dios y otras no.

La paradoja aquí señalada pone en duda la armonía de la teoría cartesiana de la idea de Dios, especialmente si se lo concibe como absolutamente perfecto, bueno y benevolente, en relación con el libre albedrío y el error humano. Se mostró como, al final, Dios no podría ser definido como un ser que contiene todo los demás seres y categorías imaginables e inimaginables y que su creación "el universo" deba ser entendido como una perfección de tipo no equiparable a la de Dios. En otras palabras, es necesario implementar la teoría de los tipos lógicos de Rusell a estos conceptos.

Tal vez el problema se pueda resolver al delimitar la siguiente frase: "Con el nombre de Dios entiendo cierta sustancia infinita, independiente, supremamente inteligente, supremamente poderosa, por quien, tanto yo mismo, como todo lo demás que existe, si algo más existe, hemos sido creados" (Descartes, 2009, p.117). Oración problemática por la definición del conjunto infinito contenedor de todos los demás conjuntos infinitos como lo es "la creación" perfecta por la benevolencia de Dios. Dado que, de manera similar, a la paradoja de Cantor y Burali-Forti, este conjunto presenta problemas de límites difusos y puede caer en la paradoja aquí enunciada. 
Una reelaboración de la anterior frase que evadiría tal antinomia es: con el nombre de Dios entiendo cierta sustancia infinita de grado " $n$ ", independiente, supremamente inteligente, supremamente poderosa, por quien, tanto yo mismo, como todo lo demás de grado " $n$ " que existe, si algo más existe, hemos sido creados. En este escenario hipotético se evitaría la comparación del conjunto infinito en grado "n" de "Dios" con otros conjuntos infinitos de diferentes grados como lo puede ser el conjunto de la creación, excluyendo asíla posible adición o comparación. Esta pequeña delimitación del grado o tipo del conjunto, no es incluida ya que, al parecer, el concepto definido "Dios" se pretende construir sin límites; propósito que encuentra problemas al definir, en un mismo discurso, dos distintos conceptos con esta característica.

Ahora bien, como lo señala Dorter (1973), una de las razones para dudar de la sinceridad de la prueba de la existencia de Dios de Descartes tiene que ver con el lenguaje usado por Descartes, el cual es notablemente "anti-cartesiano". En efecto, en las Meditaciones Descartes ha insistido constantemente en la claridad, evidencia y simplicidad como los elementos más importantes para determinar la verdad de algo. Para evitar cualquier error es importante ir lentamente y ser cuidadosos con términos que no han sido claramente explicados y entendidos. Para esto las matemáticas, según Descartes, proporcionan un modelo ejemplar. Sin embargo, como lo afirma Dorter, tan pronto llegamos a la prueba de la existencia de Dios,

(...) these principles of clarity and distinctness are abandoned. Instead we are deluged with the whole apparatus of technical scholasticism, without a single explanation. Whereas before he found "rational animal" too opaque for his method, he now uncritically employs such terms as "substance", "objective reality", "actual reality", "formal reality", "participation by representation", "degrees of being", "degrees of perfection", "modes", "accidents", "formal causality", "eminent causality", "material truth", "material falsity”, etc., without definition, let alone inquiry as to whether they signify anything real. Far from being clear and distinct, the proof is obscure and confusing, despite the fact that it is essentially rather simple and could easily have been stated in clear terms. The style and language of the proof seem so out of character with the general procedure of the Meditations, that it is easy to believe that it is not part of the fabric of the whole, and was written from a different position than the rest of the work. (p. 327-328).

En este artículo, siendo fieles a los principios de claridad y distinción de la filosofía cartesiana, quisimos aplicar elementos matemáticos a la argumentación de 
Descartes. El resultado puede servir para confirmar las sospechas de Dorter, o simplemente para afirmar que existe una paradoja y una contradicción insalvable en la perspectiva de Descartes. Dadas las grandes habilidades matemáticas del filósofo, nos sentimos más inclinados a afirmar lo primero. Con nuestro texto quisimos entonces sumarnos a las voces no dominantes de Caton y Dorter quienes proponen provocadores argumentos en la misma dirección.

\section{REFERENCIAS}

Abbruzzese, J.E. (2008). Do Descartes and St. Thomas Agree on the Ontological Proof?. Pacific Philosophical Quarterly, Vol. 89 Issue 4, 413435. 23.

Abbagnano, N. (1994). Historia de la filosofía volumen 1. Trad. de Juan Estelrich y J. Pérez Ballestar. Barcelona: Hora, S.A.

Agostini, I. (2015). Descartes's Proofs of God and the Crisis of Thomas Aquinas's Five Ways in Early Modern Thomism: Scholastic and Cartesian Debates. Harvard Theological Review, Vol. 108 Issue 2, 235262, 28.

Aristóteles (1994). Metafísica. Libro XIII. Trad. Tomás Calvo Martínez. Madrid: Gredos.

Barwise, J. y Etchemendy, J. (1987). The Liar: An Essay on Truth and Circularity, Oxford.

Beall, J.C. (editor) (2007)., Revenge of the Liar. New Essays on the Paradox, New York: Oxford University Press.

Bolton, R. K. (2008). Descartes and the "Mystical Foundation" of the Reason (Introduction to the problem of God in the Metaphysical Meditations). Límite, Vol. 3 Issue 18, 21-46. 26.

Cantini, A., "On a Russellian paradox about propositions and truth", in: G. Link 2004, 259-284.

Cantor, G. (1915). Contribution of the Founding of the Theory of Transfinite Numbers. Traducción al inglés por Philip E. B. Jourdain. New York: Dover publication. 
Crumplin, M. (2008). Descartes: God as the Idea of Infinity. International Journal of Systematic Theology, Vol. 10 Issue 1, 3-20. 18.

Dehart, P. (2002). The Ambiguous Infinite: Jüngel, Marion, and the God of Descartes. The Journal of Religion, Vol. 82, Issue 1, 75-96.

Della Rocca, M. (2005). Descartes, the Cartesian Circle, and Epistemology without God. Philosophy and Phenomenological Research, Vol. 70, Issue $1,1-33$.

Descartes, R. (2011). Reglas para la dirección del espíritu. Trad. Luis Villoro. Madrid: Gredos.

Descartes, R. (2009). Meditaciones acerca de la filosofía primera: en la cual se demuestra la existencia de dios y la inmortalidad del alma. Trad. Jorge Aurelio Díaz. Bogotá: Universidad Nacional de Colombia.

Descartes, R. (1981). Discurso del método, dióptrica, meteros y geometría. Trad. Guillermo Quintás Alonso. Madrid: Alfaguara.

Dorter, K. (1973). Science and Religion in Descartes Meditations. The Thomist, 37 (2):313-340.

Echevarría, J. (2013). La influencia de las matemáticas en la emergencia de la filosofía moderna. En: Del Renacimiento a la Ilustración I, Trotta.

Field, H. (2008). Saving Truth from Paradox, New York: Oxford University Press.

Garber, D. (2013). God, Laws, and the Order of Nature: Descartes and Leibniz, Hobbes and Spinoza. Editor Watkins, Eric. The Divine Order, the Human Order, and the Order of Nature: Historical Perspectives, Oxford University Press.

Garciadiego, A., (1992). Bertrand Russell and the Origins of Set-theoretic “Paradoxes". Basel: Birkhäuser.

Gorham, G. (2012). Early Scientific Images of God: Descartes, Hobbes, and Newton. En: Turning Images in Philosophy, Science, and Religion: A New Book of Nature. Enero 19.

Habermas, J. (1982). Conocimiento e interés 
Habermas, J. (1985). "Ciencias sociales reconstructivas vs. Comprensivas”

Heidegger, M. (1985). La pregunta por la cosa. Trad. Eduardo García Belsunce y Zoltan Szankay. Barcelona: Ediciones Orbis S.A.

Hobbes, T. (2009). Tratado sobre el cuerpo. Ed. Joaquín Rodríguez Feo. Madrid: Universidad Nacional de Educación a Distancia.

Ingthorsson, R. (2013). The natural vs. The human sciences: myth, methodology and ontology. Discusiones Filosóficas 14 (22):25-41.

Kant, I. (1998). Crítica de la razón pura. Trad. Pedro Ribas. España: Alfaguara.

Kritchman, S. and Raz, R., (2011). "The surprise examination paradox and the second incompleteness theorem".

Leibniz, G.W. (1983). Nuevos ensayos sobre el entendimiento humano. Edit. J. Echeverría Ezponda. Madrid: Editora Nacional.

McGee, V., (1991). Truth, Vagueness, and Paradox: An Essay on the Logic of Truth, Indianapolis and Cambridge: Hackett Publishing.

Notices of the American Mathematical Society, 57: 1454-1458.

Organisation for Economic Co-Operation And Development (OECD). (2004). Reinventing the Social Sciences.

Platón (1993). La República. Trad. José Manuel Pabón y Manuel FernándezGaliano. Madrid: Altaya.

Reale, G. y Antiseri D. (1988). Historia del pensamiento filosófico y científico. Tomo segundo del humanismo a Kant. Barcelona: Herder.

Rengifo, M. (2004). Categorías una interpretación de la filosofía analítica. Bogotá: Universidad Externado de Colombia.

Russell, B. (1988). Introducción a la filosofía matemática. Trad. Mireia Vivas. España: Paidos.

Sainsbury. (2009). Paradoxes. Cambridge University Press.

Serrano, G. (2005). La querella en torno al silogismo 1605-1704: conocimiento versus forma lógica. Bogotá: Universidad Nacional de Colombia. 
Shen-Yuting, (1953). "The paradox of the class of all grounded sets", The Journal of Symbolic Logic, 18: 114.

Simmons, K., (1993). Universality and the Liar, New York: Cambridge University Press.

Steinmetz, G. (2005). The Politics of Method in the Human Sciences: Positivism and Its Epistemological Others. Duke University Press.

Tindemans, A. J., Verrijn-Stuart, A. A., Visser, R. P. W. (Ed). (2002). The Future of the Sciences and Humanities. Amsterdam University Press.

Whitehead y Russell, B. (1963). Principia Mathematica. London: Cambridge University. 